\title{
Nonequilibrium perturbative formalism and spectral function for the Anderson model
}

\author{
M.Hamasaki \\ Department of Physics, Kyoto University, Kyoto 606-8502, Japan \\ Received September 14, 2006, in final form December 24, 2006
}

The present work is based on the nonequilibrium perturbative formalism. There the self-energies are derived up to the fourth-order. Consequently, it proves that the nonequilibrium (real-time) perturbative expansion can be connected with the Matsubara imaginary-time perturbative expansion for equilibrium. As the numerical results, the Kondo resonance still disappears for bias voltage exceeding the Kondo temperatures, as observed in experiments of two terminal systems.

Key words: nonequilibrium perturbative formalism, nonequilibrium Green's function, Kondo effect, Dyson's equation

PACS: $71.15 .-m, 05.70 . L n$

\section{Introduction}

\subsection{Nonequilibrium perturbative formalism}

The basic idea on the nonequilibrium perturbation theory grounded on the time-contour which starts and ends at $t=-\infty$ via $t=\infty$ was proposed by Schwinger [1]. Thereafter, the frame of the nonequilibrium perturbation theory was constructed using the nonequilibrium Green's functions given after the time-contour by Keldysh [2]. The perturbative equation is expressed in matrix form:

$$
\mathbf{G}=\mathbf{g}+\mathbf{g} \Sigma \mathbf{G}
$$

where

$$
\mathbf{G}=\left[\begin{array}{ll}
G^{--} & G^{<} \\
G^{>} & G^{++}
\end{array}\right], \quad \boldsymbol{\Sigma}=\left[\begin{array}{cc}
\Sigma^{--} & \Sigma^{<} \\
\Sigma^{>} & \Sigma^{++}
\end{array}\right] .
$$

The nonequilibrium Green's functions are given in the Heisenberg representation by

$$
\begin{aligned}
G^{--}\left(t_{1}, t_{2}\right) & \equiv-\mathrm{i}\left\langle\mathrm{T} \hat{d}\left(t_{1}\right) \hat{d}^{\dagger}\left(t_{2}\right)\right\rangle, \\
G^{++}\left(t_{1}, t_{2}\right) & \equiv-\mathrm{i}\left\langle\tilde{\mathrm{T}} \hat{d}\left(t_{1}\right) \hat{d}^{\dagger}\left(t_{2}\right)\right\rangle, \\
G^{>}\left(t_{1}, t_{2}\right) & \equiv-\mathrm{i}\left\langle\hat{d}\left(t_{1}\right) \hat{d}^{\dagger}\left(t_{2}\right)\right\rangle, \\
G^{<}\left(t_{1}, t_{2}\right) & \equiv \mathrm{i}\left\langle\hat{d}^{\dagger}\left(t_{2}\right) \hat{d}\left(t_{1}\right)\right\rangle .
\end{aligned}
$$

Here, the time ordering operator $\mathrm{T}$ is arranged in chronological order and $\tilde{\mathrm{T}}$ is the countertime ordering operator which is arranged in the reverse chronological order. The angular brackets denote thermal average in nonequilibrium.

The present work is undertaken based on the nonequilibrium perturbative formalism, equation (1). The retarded and advanced self-energies up to the fourth-order are formulated. Then it is confirmed that the nonequilibrium perturbative expansion can be connected with the Matsubara imaginary-time perturbative expansion for equilibrium. 


\subsection{The Kondo effect}

The Kondo effect [3] was discovered forty years ago and later the Kondo physics was clarified from Landau's Fermi liquid theory [4], the renormalization group [5], scaling [6], etc. Besides, generalized Kondo problem, that of more than one channel or one impurity was investigated $[7,8]$. Then, the Kondo effect in electron transport through a quantum dot was predicted theoretically late in 1980s [9] and after a decade, this phenomenon was observed [10]. The Kondo effect was studied theoretically using the Anderson model and the predictions were confirmed experimentally. In the Kondo regime, the conductance was observed to reach the unitarity limit and the Kondo temperatures estimated from observation [11] are in excellent agreement with the expression derived using the Anderson model [12]. Furthermore, the Kondo effect in a quantum dot was studied for nonequilibrium system where the bias voltage is applied [13]. The Yamada-Yosida theory, [14] perturbation theory for equilibrium based on the Fermi liquid theory [4] was extended to nonequilibrium system and it was shown that for bias voltage higher than the Kondo temperatures, the Kondo resonance disappears in the spectral function with the second-order self-energy of the Anderson model [15]. This is in good agreement with the experiments of two terminal systems that it was observed that the Kondo effect is suppressed when source-drain bias voltage is comparable to or exceeds the Kondo temperatures $[16,17]$.

In the present work, using the self-energies derived up to the fourth-order, the behavior of Kondo resonance is investigated for nonequilibrium state caused by bias voltage. The numerical results still show that the Kondo resonance is broken, as supported by two-terminal experiments.

\section{Nonequilibrium perturbation theory}

\subsection{Formalism}

A thermal average can be obtained based on the nonequilibrium perturbation theory $[1,2,18-$ $22]$. It is assumed that we know only the state at $t=-\infty$, that is, initially at $t=-\infty$, the system is in equilibrium and/or in noninteracting state. The perturbation is turned on at $t=-\infty$ and is introduced adiabatically and then it is brought wholly into the system at $t=0$; around $t=0$, the system is regarded as stationary nonequilibrium and/or interacting state. Here after, the perturbation is taken away adiabatically and vanishes at $t=\infty$. When the time evolution of the state is irreversible, then, the state at $t=\infty$ cannot be well-defined. Therefore, the time evolution is performed along the real-time contour which starts and ends at $t=-\infty$, as illustrated in figure 1.

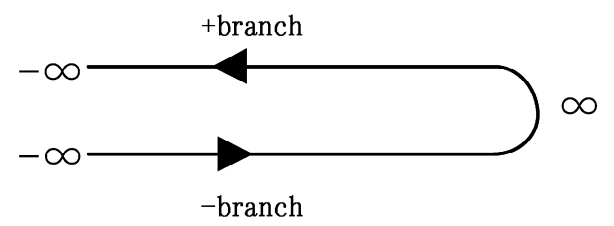

Figure 1. The time-contour which starts and ends at $t=-\infty$.

S matrix is defined by

$$
\begin{aligned}
\mathcal{S}\left(t, t_{0}\right) & =1+\sum_{n=1}^{\infty} \frac{1}{n !}\left(\frac{-\mathrm{i}}{\hbar}\right)^{n} \int_{t_{0}}^{t} \mathrm{~d} t_{1} \ldots \int_{t_{0}}^{t} \mathrm{~d} t_{n} \mathrm{~T}\left[\tilde{\mathcal{H}}_{\mathrm{I}}\left(t_{1}\right) \ldots \tilde{\mathcal{H}}_{\mathrm{I}}\left(t_{n}\right)\right] \\
& =\mathrm{T}\left[\exp \left\{\frac{-\mathrm{i}}{\hbar} \int_{t_{0}}^{t} \mathrm{~d} t^{\prime} \tilde{\mathcal{H}}_{\mathrm{I}}\left(t^{\prime}\right)\right\}\right], \\
\mathcal{S}\left(t, t_{0}\right)^{\dagger} & =\mathcal{S}\left(t_{0}, t\right)=\tilde{\mathrm{T}}\left[\exp \left\{\frac{\mathrm{i}}{\hbar} \int_{t_{0}}^{t} \mathrm{~d} t^{\prime} \tilde{\mathcal{H}}_{\mathrm{I}}\left(t^{\prime}\right)\right\}\right] .
\end{aligned}
$$


Here $\tilde{\mathcal{H}}_{\mathrm{I}}$ is perturbation term in interaction representation.

For thermal equilibrium, the statistical operator (density matrix ) is written in Gibbs form for the grand canonical ensemble by

$$
\varrho_{\mathrm{G}}=\frac{\mathrm{e}^{-\beta(\mathcal{H}-\mu N)}}{\operatorname{Tr}^{-\beta(\mathcal{H}-\mu N)}}=\mathrm{e}^{\beta(\Omega-\mathcal{H}+\mu N)} .
$$

Equation (8) is not valid exactly for nonequilibrium. We have no specific limitations upon the statistical operator. The statistical operator can generally be expressed in Schrödinger representation by $[20,22]$

$$
\varrho_{\mathrm{S}}(t)=\sum_{m}\left|m_{\mathrm{S}}(t)>P_{m}<m_{\mathrm{S}}(t)\right|
$$

Here, $P_{m}$ is probability that the system is in state $m$ and $\mid m_{\mathrm{S}}(t)>$ is the state in Schrödinger representation. $\varrho_{\mathrm{S}}$ satisfies the Liouville equation by

$$
\mathrm{i} \hbar \frac{\partial \varrho_{\mathrm{S}}}{\partial t}=\left[\mathcal{H}, \varrho_{\mathrm{S}}\right]
$$

The statistical operator in the interaction representation is given by $\tilde{\varrho}(t)=\mathrm{e}^{\mathrm{i} \mathcal{H}_{0} t / \hbar} \varrho_{\mathrm{S}}(t) \mathrm{e}^{-\mathrm{i} \mathcal{H}_{0} t / \hbar}$ and satisfies

$$
\mathrm{i} \hbar \frac{\partial \tilde{\varrho}}{\partial t}=\left[\tilde{\mathcal{H}}_{\mathrm{I}}, \tilde{\varrho}\right]
$$

As a matter of course, $\varrho_{\mathrm{S}}(0)=\varrho(0)=\tilde{\varrho}(0)$. Here $\varrho(t)$ is in the Heisenberg representation. The time evolution is expressed using $\mathrm{S}$ matrix by

$$
\tilde{\varrho}(t)=S\left(t, t_{0}\right) \tilde{\varrho}\left(t_{0}\right) S\left(t_{0}, t\right) .
$$

The thermal average in the Heisenberg representation at $t=0$ can be obtained, for example by $[18,19,22]$

$$
\begin{aligned}
\left\langle\mathrm{T} A(t) B\left(t^{\prime}\right)\right\rangle \equiv & \operatorname{Tr}\left[\varrho(0) \mathrm{T} A(t) B\left(t^{\prime}\right)\right]=\operatorname{Tr}\left[\tilde{\varrho}(-\infty) \mathcal{S}(-\infty, 0) \mathrm{T} A(t) B\left(t^{\prime}\right) \mathcal{S}(0,-\infty)\right] \\
= & \operatorname{Tr}\left[\tilde{\varrho}(-\infty) \mathcal{S}(-\infty, \infty)\left\{\mathrm{T} \mathcal{S}(\infty,-\infty) \tilde{A}\left(t^{-}\right) \tilde{B}\left(t^{\prime}-\right)\right\}\right] \\
= & \sum_{n=1}^{\infty} \sum_{m=1}^{\infty} \frac{1}{n !} \frac{1}{m !}\left(\frac{\mathrm{i}}{\hbar}\right)^{n}\left(\frac{-\mathrm{i}}{\hbar}\right)^{m} \int_{-\infty}^{\infty} \mathrm{d} t_{1} \ldots \int_{-\infty}^{\infty} \mathrm{d} t_{n} \int_{-\infty}^{\infty} \mathrm{d} t_{1}^{\prime} \ldots \int_{-\infty}^{\infty} \mathrm{d} t_{m}^{\prime} \\
& \times\left\langle\left\{\tilde{\mathrm{T}} \tilde{\mathcal{H}}_{\mathrm{I}}\left(t_{1}^{+}\right) \ldots \tilde{\mathcal{H}}_{\mathrm{I}}\left(t_{n}^{+}\right)\right\}\left\{\mathrm{T} \tilde{\mathcal{H}}_{\mathrm{I}}\left(t_{1}^{\prime}\right) \ldots \tilde{\mathcal{H}}_{\mathrm{I}}\left(t_{m}^{\prime}\right) \tilde{A}\left(t^{-}\right) \tilde{B}\left(t^{\prime}-\right)\right\}\right\rangle_{\mathrm{av}},
\end{aligned}
$$

where $\langle\ldots\rangle_{\mathrm{av}}=\operatorname{Tr}[\tilde{\varrho}(-\infty) \ldots]$. Then, the thermal average is derived by following the ordinary procedure via the Wick's theorem.

\subsection{Relation of self-energy}

After the perturbative expansion is executed, the retarded and advanced self-energies are formulated. According to the definition, the retarded and advanced Green's functions are given by

$$
\begin{aligned}
& G^{r}\left(t_{1}, t_{2}\right) \equiv-\mathrm{i} \theta\left(t_{1}-t_{2}\right)\left\langle\left\{\hat{d}\left(t_{1}\right), \hat{d}^{\dagger}\left(t_{2}\right)\right\}\right\rangle, \\
& G^{a}\left(t_{1}, t_{2}\right) \equiv \mathrm{i} \theta\left(t_{2}-t_{1}\right)\left\langle\left\{\hat{d}\left(t_{1}\right), \hat{d}^{\dagger}\left(t_{2}\right)\right\}\right\rangle .
\end{aligned}
$$

Here, the curly brackets denote anticommutator. The Dyson's equations for the retarded and advanced Green's functions are given by

$$
\begin{aligned}
G^{r} & =g^{r}+g^{r} \Sigma^{r} G^{r}, \\
G^{a} & =g^{a}+g^{a} \Sigma^{a} G^{a} .
\end{aligned}
$$


As the necessity to equations (15) and (16), the self-energies $\Sigma^{r}$ and $\Sigma^{a}$ are also required to be retarded and advanced functions in time, respectively. In accordance with the ordinary procedure of nonequilibrium perturbative formalism, $[2,19]$ there

$$
\mathbf{L}=\left[\mathbf{L}^{\dagger}\right]^{-1}=\frac{1}{\sqrt{2}}\left[\begin{array}{ll}
1 & -1 \\
1 & 1
\end{array}\right]
$$

and using this, then,

$$
\boldsymbol{\Sigma}=\left[\begin{array}{ll}
\Sigma^{--} & \Sigma^{<} \\
\Sigma^{>} & \Sigma^{++}
\end{array}\right] \longrightarrow \mathbf{L} \boldsymbol{\Sigma}^{\dagger}=\left[\begin{array}{ll}
\Omega & \Sigma^{r} \\
\Sigma^{a} & 0
\end{array}\right]
$$

The relationship for self-energies ought to be obtained here by comparison of equation (1) with equations (15) and (16):

$$
\begin{aligned}
& \Sigma^{r}(t)=\Sigma^{--}(t)+\Sigma^{<}(t)=-\Sigma^{++}(t)-\Sigma^{>}(t) \\
& \Sigma^{a}(t)=\Sigma^{--}(t)+\Sigma^{>}(t)=-\Sigma^{++}(t)-\Sigma^{<}(t) .
\end{aligned}
$$

\section{Expressions of self-energy for Anderson model}

\subsection{Anderson model}

We consider equilibrium and nonequilibrium stationary states. Nonequilibrium state is caused by finite bias voltage, that is, the difference of chemical potentials; after bias voltage was turned on, long time has passed enough to reach stationary states. Since the states are stationary, Hamiltonian has no time dependence. The system is described by the Anderson model connected to leads. The impurity with on-site energy $E_{0}$ and the Coulomb interaction $U$ is connected to the left and right leads by the mixing matrix elements, $v_{\mathrm{L}}$ and $v_{\mathrm{R}}$. The Anderson Hamiltonian is given by

$$
\begin{aligned}
\mathcal{H}=\quad & E_{0} \sum_{\sigma} \hat{n}_{d \sigma}+\mu_{\mathrm{L}} \sum_{\sigma} \hat{n}_{L \sigma}+\mu_{\mathrm{R}} \sum_{\sigma} \hat{n}_{R \sigma}+U\left(\hat{n}_{d \uparrow}-\left\langle\hat{n}_{d \uparrow}\right\rangle\right)\left(\hat{n}_{d \downarrow}-\left\langle\hat{n}_{d \downarrow}\right\rangle\right) \\
& -\sum_{\sigma} v_{\mathrm{L}}\left(\hat{d}_{\sigma}^{\dagger} \hat{c}_{L \sigma}+\text { H.c. }\right)-\sum_{\sigma} v_{\mathrm{R}}\left(\hat{d}_{\sigma}^{\dagger} \hat{c}_{R \sigma}+\text { H.c. }\right) .
\end{aligned}
$$

$\hat{d}^{\dagger}(\hat{d})$ is creation (annihilation) operator for electron on the impurity, and $\hat{c}_{\mathrm{L}}^{\dagger}$ and $\hat{c}_{\mathrm{R}}^{\dagger}\left(\hat{c}_{\mathrm{L}}\right.$ and $\left.\hat{c}_{\mathrm{R}}\right)$ are creation (annihilation) operators in the left and right leads, respectively. $\sigma$ is index for spin. The chemical potentials in the isolated left and right leads are $\mu_{\mathrm{L}}$ and $\mu_{\mathrm{R}}$, respectively. The applied voltage is, therefore defined by $\mathrm{eV} \equiv \mu_{\mathrm{L}}-\mu_{\mathrm{R}}$.

We consider that the band-width of left and right leads is infinitely large, so that the coupling functions, $\Gamma_{\mathrm{L}}$ and $\Gamma_{\mathrm{R}}$ can be taken to be independent of energy, $E$. On-site energy $E_{0}$ is set cancelling with the Hartree term, i.e. the first-order contribution to self-energy for electron correlation: $\Sigma_{\sigma}^{r(1)}(E)=\Sigma_{\sigma}^{a(1)}(E)=U\left\langle n_{-\sigma}\right\rangle$.

Accordingly, the Fourier components of the noninteracting (unperturbed) Green's functions reduce to

$$
\begin{aligned}
& g^{r}(E)=\frac{1}{E+\mathrm{i} \Gamma}, \\
& g^{a}(E)=\frac{1}{E-\mathrm{i} \Gamma},
\end{aligned}
$$

where $\Gamma=\left(\Gamma_{L}+\Gamma_{R}\right) / 2$. Hence, the inverse Fourier components can be written by

$$
\begin{aligned}
g^{r}(t) & =-\mathrm{i} \theta(t) \mathrm{e}^{-\Gamma t} \\
g^{a}(t) & =\mathrm{i} \theta(-t) \mathrm{e}^{\Gamma t}
\end{aligned}
$$


Moreover, from equation (1), we have

$$
\begin{aligned}
& g^{<}(E)=g^{r}(E)\left[\mathrm{i} f_{\mathrm{L}}(E) \Gamma_{\mathrm{L}}+\mathrm{i} f_{\mathrm{R}}(E) \Gamma_{\mathrm{R}}\right] g^{a}(E), \\
& g^{>}(E)=g^{r}(E)\left[\mathrm{i}\left(f_{\mathrm{L}}(E)-1\right) \Gamma_{\mathrm{L}}+\mathrm{i}\left(f_{\mathrm{R}}(E)-1\right) \Gamma_{\mathrm{R}}\right] g^{a}(E) .
\end{aligned}
$$

$f_{\mathrm{L}}$ and $f_{\mathrm{R}}$ are the Fermi distribution functions in the isolated left and right leads, respectively. By equations (22) and (23), the nonequilibrium state is introduced as the superposition of the left and right leads. Then, the effective Fermi distribution function can be expressed by [15]

$$
f_{\mathrm{eff}}(E)=\frac{f_{\mathrm{L}}(E) \Gamma_{\mathrm{L}}+f_{\mathrm{R}}(E) \Gamma_{\mathrm{R}}}{\Gamma_{\mathrm{L}}+\Gamma_{\mathrm{R}}} .
$$

\subsection{Self-energy}

The retarded and advanced self-energies are derived up to the fourth-order. Equations (17) and (18) are divided into retarded and advanced terms in time:

$$
\begin{aligned}
\Sigma^{r}(t) & =\left[\Sigma^{--}(t)+\Sigma^{<}(t)\right] \theta(t)+\left[\Sigma^{--}(t)+\Sigma^{<}(t)\right] \theta(-t) \\
& =-\left[\Sigma^{++}(t)+\Sigma^{>}(t)\right] \theta(t)-\left[\Sigma^{++}(t)+\Sigma^{>}(t)\right] \theta(-t), \\
\Sigma^{a}(t) & =\left[\Sigma^{--}(t)+\Sigma^{>}(t)\right] \theta(t)+\left[\Sigma^{--}(t)+\Sigma^{>}(t)\right] \theta(-t) \\
& =-\left[\Sigma^{++}(t)+\Sigma^{<}(t)\right] \theta(t)-\left[\Sigma^{++}(t)+\Sigma^{<}(t)\right] \theta(-t) .
\end{aligned}
$$

Then it is found that for the self-energies drawn using the Wick's theorem,

$$
\begin{aligned}
\Sigma^{--}(t) \theta(t) & =-\Sigma^{>}(t) \theta(t), & & \Sigma^{++}(t) \theta(t)=-\Sigma^{<}(t) \theta(t), \\
\Sigma^{--}(t) \theta(-t) & =-\Sigma^{<}(t) \theta(-t), & & \Sigma^{++}(t) \theta(-t)=-\Sigma^{>}(t) \theta(-t) .
\end{aligned}
$$

This leads to

$$
\begin{aligned}
{\left[\Sigma^{--}(t)+\Sigma^{<}(t)\right] \theta(-t) } & =-\left[\Sigma^{++}(t)+\Sigma^{>}(t)\right] \theta(-t)=0 \\
{\left[\Sigma^{--}(t)+\Sigma^{>}(t)\right] \theta(t) } & =-\left[\Sigma^{++}(t)+\Sigma^{<}(t)\right] \theta(t)=0 .
\end{aligned}
$$

As a consequence, the retarded and advanced self-energies are obtained as retarded and advanced functions of time, respectively:

$$
\begin{aligned}
& \Sigma^{r}(t)=\left[\Sigma^{--}(t)+\Sigma^{<}(t)\right] \theta(t)=-\left[\Sigma^{++}(t)+\Sigma^{>}(t)\right] \theta(t), \\
& \Sigma^{a}(t)=\left[\Sigma^{--}(t)+\Sigma^{>}(t)\right] \theta(-t)=-\left[\Sigma^{++}(t)+\Sigma^{<}(t)\right] \theta(-t) .
\end{aligned}
$$

Moreover, it proves

$$
\begin{aligned}
& \Sigma^{r}(t)=\left[\Sigma^{<}(t)-\Sigma^{>}(t)\right] \theta(t), \\
& \Sigma^{a}(t)=\left[\Sigma^{>}(t)-\Sigma^{<}(t)\right] \theta(-t) .
\end{aligned}
$$

Hence the following relations still stand: $\Sigma^{r}-\Sigma^{a}=\Sigma^{<}-\Sigma^{>}$and furthermore

$$
\begin{aligned}
& G^{<}=\left(1+G^{r} \Sigma^{r}\right) g^{<}\left(1+G^{a} \Sigma^{a}\right)-G^{r} \Sigma^{<} G^{a}, \\
& G^{>}=\left(1+G^{r} \Sigma^{r}\right) g^{>}\left(1+G^{a} \Sigma^{a}\right)-G^{r} \Sigma^{>} G^{a} .
\end{aligned}
$$

As the result, the second-order self-energy is written by

$$
\begin{aligned}
\Sigma^{r(2)}(E) & =U^{2} \int_{0}^{\infty} \mathrm{d} t_{1} \mathrm{e}^{\mathrm{i} E t_{1}}\left[\begin{array}{c}
g^{>}\left(t_{1}\right) g^{>}\left(t_{1}\right) g^{<}\left(-t_{1}\right) \\
-g^{<}\left(t_{1}\right) g^{<}\left(t_{1}\right) g^{>}\left(-t_{1}\right)
\end{array}\right] \\
& =U^{2} \int_{0}^{\infty} \mathrm{d} t_{1} \mathrm{e}^{\mathrm{i} E t_{1}}\left[\begin{array}{c}
g^{ \pm}\left(t_{1}\right) g^{>}\left(t_{1}\right) g^{<}\left(-t_{1}\right) \\
+g^{<}\left(t_{1}\right) g^{ \pm}\left(t_{1}\right) g^{>}\left(-t_{1}\right) \\
+g^{<}\left(t_{1}\right) g^{>}\left(t_{1}\right) g^{ \pm}\left(-t_{1}\right)
\end{array}\right],
\end{aligned}
$$




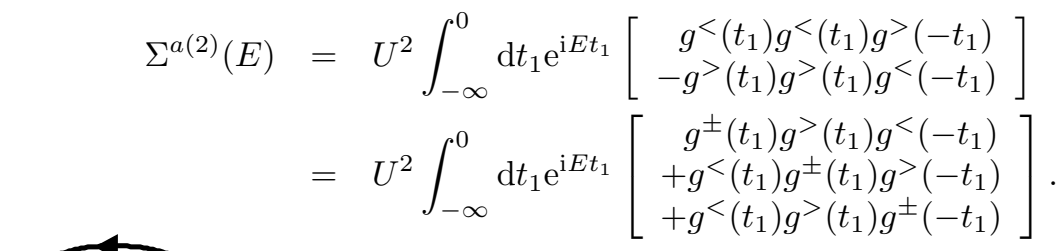

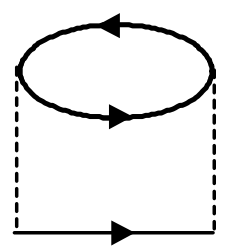

Figure 2. The diagram for the second-order self-energy. The solid line denotes the noninteracting Green's function and the dashed line denotes interaction.
Here $g^{ \pm}(t)=g^{r}(t)+g^{a}(t)$, that is, $g^{+}(t)=g^{r}(t)=-\mathrm{i} \theta(t) \mathrm{e}^{-\Gamma t}$ for $t \geqslant 0$ and $g^{-}(t)=g^{a}(t)=\mathrm{i} \theta(-t) \mathrm{e}^{\Gamma t}$ for $t<0$. Moreover, $g^{<}(t)$ and $g^{>}(t)$ are the inverse Fourier components of equations (22) and (23). Figure 2 shows the diagram for the second-order selfenergy. As shown numerically later, the second-order contribution coincides with those derived by Hershfield et al. [15]. In the symmetric equilibrium case, the asymptotic behavior at low energy is expressed by

$$
\Sigma^{r(2)}(E) \simeq-\Gamma\left(3-\frac{\pi^{2}}{4}\right)\left(\frac{U}{\pi \Gamma}\right)^{2} \frac{E}{\Gamma}-i \frac{\Gamma}{2}\left(\frac{U}{\pi \Gamma}\right)^{2}\left(\frac{E}{\Gamma}\right)^{2},
$$

the exact results are based on the Bethe ansatz method [23,24].

The third-order terms corresponding to the diagram in figure $3 \mathrm{a}$ are expressed by

$$
\begin{aligned}
\Sigma_{p p}^{r(3)}(E)= & U^{3} \int_{0}^{\infty} \mathrm{d} t_{1} \int_{-\infty}^{\infty} \mathrm{d} t_{2} \mathrm{e}^{\mathrm{i} E t_{1}}\left[\begin{array}{c}
g^{<}\left(-t_{1}\right) g^{>}\left(t_{1}-t_{2}\right) g^{>}\left(t_{1}-t_{2}\right) \\
-g^{>}\left(-t_{1}\right) g^{<}\left(t_{1}-t_{2}\right) g^{<}\left(t_{1}-t_{2}\right)
\end{array}\right] \\
& \times\left[g^{ \pm}\left(t_{2}\right) g^{>}\left(t_{2}\right)+g^{<}\left(t_{2}\right) g^{ \pm}\left(t_{2}\right)\right], \\
\Sigma_{p p}^{a(3)}(E)= & U^{3} \int_{-\infty}^{0} \mathrm{~d} t_{1} \int_{-\infty}^{\infty} \mathrm{d} t_{2} \mathrm{e}^{\mathrm{i} E t_{1}}\left[\begin{array}{c}
g^{>}\left(-t_{1}\right) g^{<}\left(t_{1}-t_{2}\right) g^{<}\left(t_{1}-t_{2}\right) \\
-g^{<}\left(-t_{1}\right) g^{>}\left(t_{1}-t_{2}\right) g^{>}\left(t_{1}-t_{2}\right)
\end{array}\right] \\
& \times\left[g^{ \pm}\left(t_{2}\right) g^{>}\left(t_{2}\right)+g^{<}\left(t_{2}\right) g^{ \pm}\left(t_{2}\right)\right] .
\end{aligned}
$$

Figure $3 \mathrm{~b}$ illustrates the diagram for the following terms:

$$
\begin{aligned}
\Sigma_{p h}^{r(3)}(E)= & U^{3} \int_{0}^{\infty} \mathrm{d} t_{1} \int_{-\infty}^{\infty} \mathrm{d} t_{2} \mathrm{e}^{\mathrm{i} E t_{1}}\left[\begin{array}{c}
g^{>}\left(t_{1}\right) g^{>}\left(t_{1}-t_{2}\right) g^{<}\left(t_{2}-t_{1}\right) \\
-g^{<}\left(t_{1}\right) g^{<}\left(t_{1}-t_{2}\right) g^{>}\left(t_{2}-t_{1}\right)
\end{array}\right] \\
& \times\left[g^{ \pm}\left(t_{2}\right) g^{<}\left(-t_{2}\right)+g^{<}\left(t_{2}\right) g^{ \pm}\left(-t_{2}\right)\right], \\
\Sigma_{p h}^{a(3)}(E)= & U^{3} \int_{-\infty}^{0} \mathrm{~d} t_{1} \int_{-\infty}^{\infty} \mathrm{d} t_{2} \mathrm{e}^{\mathrm{i} E t_{1}}\left[\begin{array}{c}
g^{<}\left(t_{1}\right) g^{<}\left(t_{1}-t_{2}\right) g^{>}\left(t_{2}-t_{1}\right) \\
-g^{>}\left(t_{1}\right) g^{>}\left(t_{1}-t_{2}\right) g^{<}\left(t_{2}-t_{1}\right)
\end{array}\right] \\
& \times\left[g^{ \pm}\left(t_{2}\right) g^{<}\left(-t_{2}\right)+g^{<}\left(t_{2}\right) g^{ \pm}\left(-t_{2}\right)\right] .
\end{aligned}
$$

Equations (28)-(31) for equilibrium agree exactly with those derived from the Matsubara imaginarytime perturbative expansion for equilibrium and analytical continuity by Zlatić et al. [25]. As mentioned later, it is numerically confirmed that the third-order contribution vanishes for the symmetric Anderson model; this is in good agreement with both the results derived from the Yamada-Yosida theory $[14,24,26]$ and obtained based on the Bethe ansatz method [23].

Furthermore, the fourth-order contribution to the self-energy is formulated (see Appendix). The twelve terms for the proper fourth-order self-energy can be divided into four groups, each of which comprises three terms. The four groups correspond to the diagrams denoted in figures $4 \mathrm{a}-\mathrm{c}$, figures $4 \mathrm{~d}-\mathrm{f}$, figures $4 \mathrm{~g}-\mathrm{i}$, and figures $4 \mathrm{j}-\mathrm{l}$, respectively. For symmetric Anderson model at equilibrium, the asymptotic behavior at low energy is approximately in agreement with those based on the Bethe ansatz method [23]:

$$
\Sigma^{r(4)}(E) \simeq-\Gamma\left(105-\frac{45 \pi^{2}}{4}+\frac{\pi^{4}}{16}\right)\left(\frac{U}{\pi \Gamma}\right)^{4} \frac{E}{\Gamma}-\mathrm{i} \frac{\Gamma}{2}\left(30-3 \pi^{2}\right)\left(\frac{U}{\pi \Gamma}\right)^{4}\left(\frac{E}{\Gamma}\right)^{2} .
$$




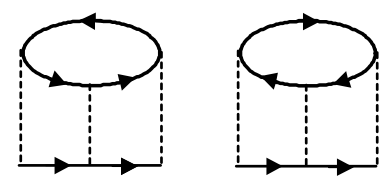

Figure 3. The two diagrams for the third-order self-energy. Left: (a) and Right: (b)
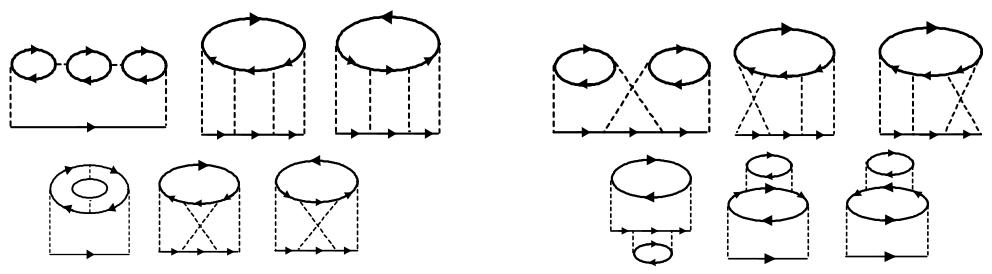

Figure 4. The twelve terms for the proper fourth-order self-energy divided into four groups: (a)-(c), (d)-(f), (g)-(i), and (j)-(l).

\section{Numerical results and discussion}

\subsection{Self-energy}

The third-order terms, equations (28)-(31) cancel under electron-hole symmetry not only at equilibrium but also at nonequilibrium: $\Sigma_{p h}^{r(3)}(E)=-\Sigma_{p p}^{r(3)}(E)$ and $\Sigma_{p h}^{a(3)}(E)=-\Sigma_{p p}^{a(3)}(E)$. As a consequence, the third-order contribution to self-energy vanishes in the symmetric case. It agrees with the results of $[14,24,26]$ based on the Yamada-Yosida theory that all odd-order contributions except the Hartree term vanish for equilibrium in the symmetric single-impurity Anderson model; probably, it is just the same with nonequilibrium state. On the other hand, the third-order terms contribute to the asymmetric system where electron-hole symmetry breaks and furthermore, the third-order terms for spin-up and for spin-down contribute respectively when the spin degeneracy is lifted for example, by magnetic field. For the fourth-order contribution, three terms which constitute each of the four groups contribute equivalently under electron-hole symmetry. Moreover, to the asymmetric system, the terms brought by the diagrams of figures $4 \mathrm{a}$ and $4 \mathrm{~b}$ contribute equivalently and the terms by the diagrams of figures $4 \mathrm{j}$ and $4 \mathrm{k}$ make equivalent contribution, and the rest, the eight terms contribute respectively. Further, the twenty-four terms for spin-up and spin-down take effect severally in the presence of magnetic field.

The second-order and the fourth-order contributions to self-energy for zero temperature symmetric Anderson model are shown in figures $5 \mathrm{a}$ and $5 \mathrm{~b}$ and in figures $6 \mathrm{a}$ and $6 \mathrm{~b}$, respectively. Equation (27) represents the curves around $E=0$ denoted by solid line in figures $5 \mathrm{a}$ and $5 \mathrm{~b}$, respectively, and equation (32) represents approximately those shown in figures $6 \mathrm{a}$ and $6 \mathrm{~b}$, respectively. The curves of the second-order self-energy shown in figures $5 \mathrm{a}$ and $5 \mathrm{~b}$ are identical with those of expressions derived by Hershfield et al. [15]. In comparison of figures $6 \mathrm{a}$ and $6 \mathrm{~b}$ with figures $5 \mathrm{a}$ and $5 \mathrm{~b}$, it is found that the fourth-order contribution for equilibrium has the same but narrow curves at low energy with those of the second-order contribution. In addition, the broad curves are attached at high energy for the fourth-order self-energy. (The higher the contribution is, the more should the curves oscillate as a function of energy.) When the voltage, $\mathrm{eV} / \Gamma$ exceeds $\sim 2.0$, the behavior of the curves of self-energy changes distinctly and comes to present striking contrast to the behaviour for the second-order contribution. Particularly, the curve for the imaginary part of the fourth-order contribution rises up with maximum at $E=0$. On the other hand, for the second-order contribution, a valley appears with a minimum at the energy of zero- which is quite the contrary. Moreover, from these results, it is expected that the sixth-order contribution to imaginary part of self-energy has a minimum at $E=0$. Hence, the perturbative expansion is hard to converge for $\mathrm{eV} / \Gamma>\sim 2.0$, as mentioned later. 

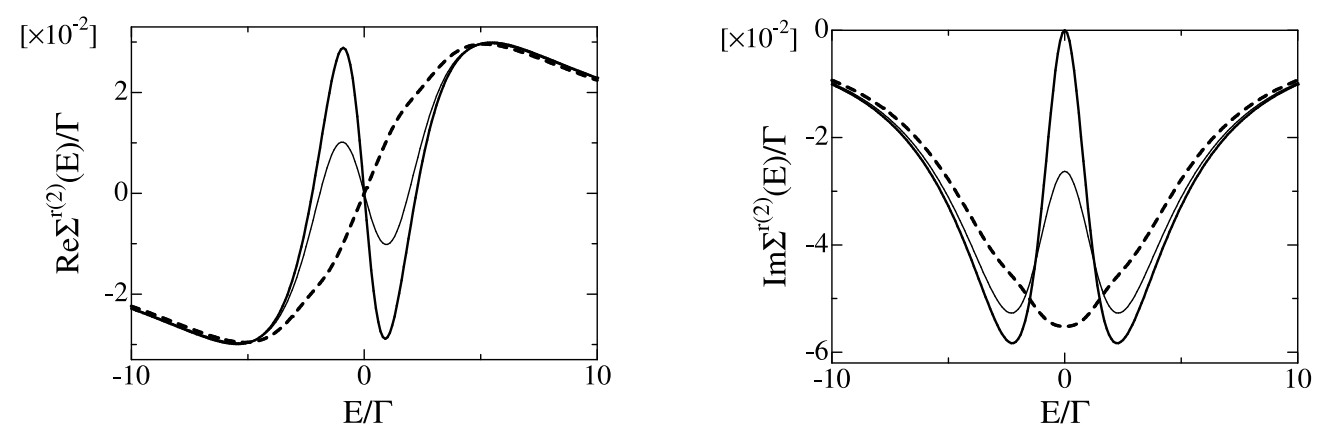

Figure 5. The second-order self-energy for the symmetric Anderson model at $U / \Gamma=1.0$ and zero temperature. (a) The real part and (b) the imaginary part at equilibrium (solid line), $\mathrm{eV} / \Gamma=1.0$ (thin solid line), and $\mathrm{eV} / \Gamma=2.0$ (dashed line).
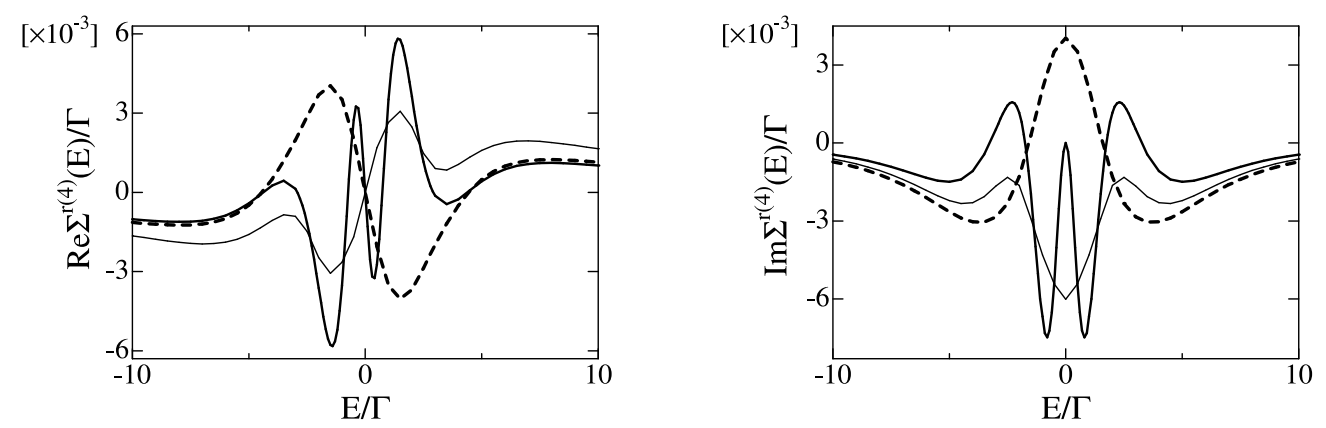

Figure 6. The fourth-order self-energy for the symmetric Anderson model at $U / \Gamma=1.0$ and zero temperature. (a) The real part and (b) the imaginary part at equilibrium (solid line), $\mathrm{eV} / \Gamma=1.0$ (thin solid line), and $\mathrm{eV} / \Gamma=2.0$ (dashed line). The fourth-order contribution for equilibrium has the same but narrow curves at low energy with those of the second-order contribution.

Besides, the current conservation is mentioned. In [15], it is shown that the continuity of current entering and leaving the impurity stands exactly at any strength of $U$ within the approximation up to the second-order for the symmetric single-impurity Anderson model. Comparing figures 6a and $6 \mathrm{~b}$ with figures $5 \mathrm{a}$ and $5 \mathrm{~b}$, it is found that the curves of the fourth-order self-energy have the symmetry similar to those of the second-order. Hence, it is anticipated that the current conservation is satisfied perfectly with approximation up to the fourth-order in the single-impurity system where electron-hole symmetry holds. The continuity of the current can be maintained perfectly in a single-impurity system as far as electron-hole symmetry stands. On the other hand, the current comes to fail to be conserved with increasing $U$ in asymmetric single-impurity case and in two-impurity case.

\subsection{Spectral function}

The spectral function with the second-order self-energy is generally known. It is plotted for $U / \Gamma=10.0$ and zero temperature in figure 7 . For equilibrium, the Kondo peak at energy of zero is very sharp and the two-side broad peaks appear at $E \simeq \pm U / 2$. The curve for $\mathrm{eV}=0$ is identical with that shown in [24]. As eV becomes higher than the Kondo temperatures, $k_{\mathrm{B}} T_{\mathrm{K}}$ [27], the Kondo peak becomes lower and finally vanishes, while the two-side broad peaks rise at $E \simeq \pm U / 2$ [15].

Figure 8 shows the spectral function with the self-energy up to the fourth-order for equilibrium and zero temperature. With strengthening $U$, two-side narrow peaks come to occur in the vicinity of $E= \pm U / 2$ in addition to the Kondo peak. At $U$ large enough, the Kondo peak becomes very acute and two-side narrow peaks rise higher and sharpen; the energy levels for the atomic limit are produced distinctly. The fourth-order self-energy has the same but narrow curves as functions of energy with those of the second-order and those curves make the peaks at $E= \pm U / 2$. 


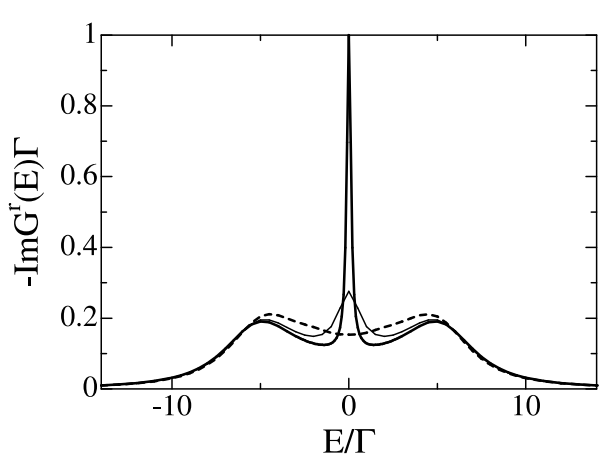

Figure 7. The spectral function with the second-order self-energy at $U / \Gamma=10.0$ for the symmetric Anderson model at equilibrium (solid line), $\mathrm{eV} / \Gamma=1.0$ (thin solid line) and $\mathrm{eV} / \Gamma=2.0$ (dashed line).

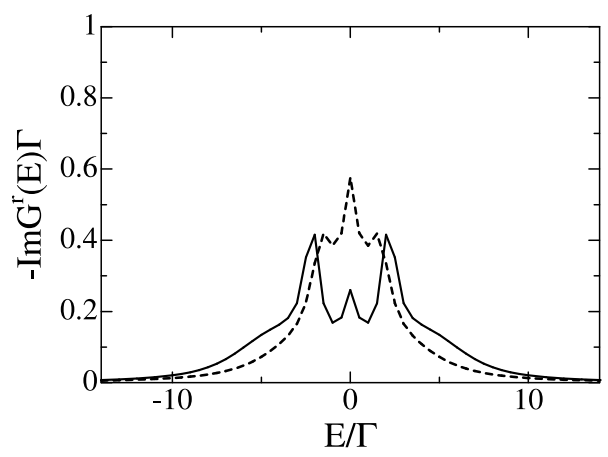

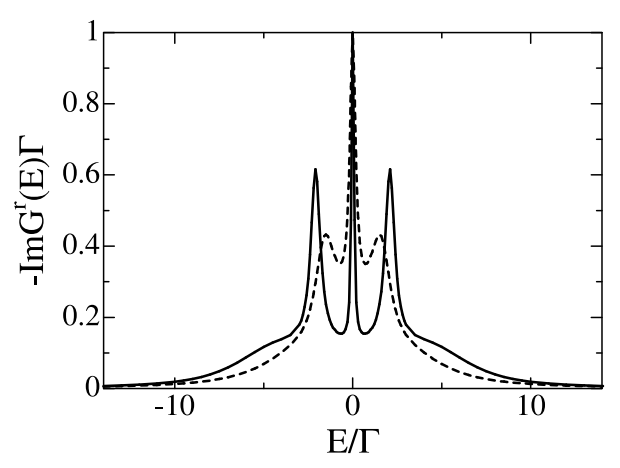

Figure 8. The spectral function with selfenergy up to the fourth-order at equilibrium for the symmetric Anderson model at $U / \Gamma=$ 3.5 (dashed line) and $U / \Gamma=5.0$ (solid line).

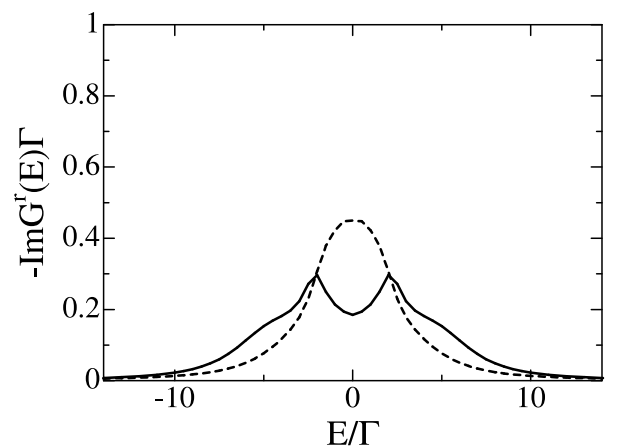

Figure 9. The spectral functions with self-energy up to the fourth-order at $\mathrm{eV} / \Gamma=0.5$ (Left) and $\mathrm{eV} / \Gamma=1.0$ (Right) for the symmetric Anderson model. $U / \Gamma=3.5$ (dashed line) and $U / \Gamma=5.0$ (solid line).

For the present approximation up to the fourth-order, the Kondo peak at $E=0$ reaches the unitarity limit and the charge, $\langle n\rangle$ corresponds to $1 / 2$, that is, the Friedel sum rule is correctly satisfied: [28]

$$
\rho\left(E_{\mathrm{f}}\right)=\sin ^{2}(\pi\langle n\rangle) / \pi \Gamma,
$$

where $\rho\left(E_{\mathrm{f}}\right)$ is the local density of states at the Fermi energy. Here, the discussions should be made concerning the ranges of $U$ in which the present approximation up to the fourth-order stands. From the results, it is found that the approximation within the fourth-order holds up to $U / \Gamma \sim 5.0$ and is beyond the validity for $U / \Gamma>\sim 6.0$. Moreover, the curve for imaginary part of the fourth-order contribution is partly positive, as shown in figure $6 \mathrm{~b}$ and as a consequence, the curve of the spectral function becomes partly negative for too large $U$. In such a case, the present approximation is out of validity and the higher-order terms are required.

Next, the results for nonequilibrium and zero temperature are shown. The expression for the Friedel sum rule, equation (33) does not stand for nonequilibrium, since the charge cannot be expressed with respect to the local density of states. All the same, the Kondo peak reaches the unitarity limit and $\langle n\rangle=1 / 2$ in the symmetric and noninteracting case. The spectral functions with the self-energy up to the fourth-order are plotted for $\mathrm{eV} / \Gamma=0.5$ and $\mathrm{eV} / \Gamma=1.0$ in figures 9 , respectively. When $U$ is strengthened and eV exceeds $k_{\mathrm{B}} T_{\mathrm{K}}$ (approximately, $k_{\mathrm{B}} T_{\mathrm{K}} / \Gamma \sim 0.5$ for $U / \Gamma$ $=3.5$ and $k_{\mathrm{B}} T_{\mathrm{K}} / \Gamma \sim 0.3$ for $U / \Gamma=5.0$ ), the Kondo peak for $\mathrm{eV} / \Gamma=0.5$ falls in and instead, the 
two-side narrow peaks remain to sharpen in the vicinity of $E= \pm U / 2$. For $\mathrm{eV} / \Gamma=1.0$, the Kondo peak becomes broad and disappears for $U$ large enough. The two-side peaks are generated small in the vicinity of $E= \pm U / 2$. The Kondo resonance is quite broken for bias voltage exceeding the Kondo temperatures; this accords with the experimental results of two terminal systems that the Kondo effect is suppressed when source-drain bias voltage is comparable to or exceeds the Kondo temperatures, $\mathrm{eV} \geqslant k_{\mathrm{B}} T_{\mathrm{K}}[16,17]$. For $\mathrm{eV} / \Gamma>\sim 2.0$, the Kondo peak does not lower even when $\mathrm{eV}$ is much larger than $k_{\mathrm{B}} T_{\mathrm{K}}$. The perturbative expansion is hard to converge on account of the imaginary part of the self-energy for $\mathrm{eV} / \Gamma>\sim 2.0$, as described before; thereby, the higher-order contribution to self-energy is probably needed for high voltage.

In the present work, nonequilibrium state is represented as the superposition of the two leads and the effective Fermi distribution function, equation (24) is qualitatively similar to that for finite temperatures. From the analogy in the Fermi distribution function, it is inferred that there are nonequilibrium fluctuations similar to thermal fluctuations [29]. Because of the effective Fermi distribution function, not only for the second-order but also for the fourth-order, the Kondo resonance is destroyed, qualitatively the same as for finite temperatures. In contrast, if the finite voltage state is expressed as two localized states, the numerical results of the Kondo peak splitting can be obtained. All the same, for two terminal systems, the Kondo resonance splitting may not take place for finite bias voltage.

\section{Summary}

The present work is based on the nonequilibrium perturbative formalism. Here the self-energies are derived and then it is indicated that the nonequilibrium (real-time) perturbative expansion can be related to the Matsubara imaginary-time perturbative expansion for equilibrium. As the numerical results, the Kondo peak disappears as bias voltage exceeding the Kondo temperatures. Because of the analogy of the effective Fermi distribution function for nonequilibrium with that for finite temperatures, the present result is qualitatively similar to that for finite temperatures. This characteristic appears in the experiments of two terminal systems.

\section{Acknowledgements}

The numerical calculations were executed at the Yukawa Institute Computer Facility. The multiple integrals were performed using the computer subroutine, MQFSRD of NUMPAC.

\section{Appendix}

The twelve terms for the fourth-order contribution can be divided into four groups, each of which is composed of three terms. The four groups are brought from the diagrams denoted in figures $4 \mathrm{a}-\mathrm{c}$, figures $4 \mathrm{~d}-\mathrm{f}$, figures $4 \mathrm{~g}-\mathrm{i}$, and figures $4 \mathrm{j}-\mathrm{l}$, respectively. The terms for the diagrams illustrated in figures $4 \mathrm{a}$ and $4 \mathrm{~b}$ are equivalent except for the spin indices and are expressed by

$$
\begin{aligned}
\Sigma_{a, b}^{r(4)}(E)= & U^{4} \int_{0}^{\infty} \mathrm{d} t_{1} \int_{-\infty}^{\infty} \mathrm{d} t_{2} \int_{-\infty}^{\infty} \mathrm{d} t_{3} \mathrm{e}^{\mathrm{i} E t_{1}}\left[\begin{array}{c}
g^{<}\left(t_{1}\right) g^{<}\left(t_{1}-t_{2}-t_{3}\right) g^{>}\left(-t_{1}+t_{2}+t_{3}\right) \\
-g^{>}\left(t_{1}\right) g^{>}\left(t_{1}-t_{2}-t_{3}\right) g^{<}\left(-t_{1}+t_{2}+t_{3}\right)
\end{array}\right] \\
& \times\left[g^{ \pm}\left(t_{2}\right) g^{<}\left(-t_{2}\right)+g^{<}\left(t_{2}\right) g^{ \pm}\left(-t_{2}\right)\right]\left[g^{ \pm}\left(t_{3}\right) g^{<}\left(-t_{3}\right)+g^{<}\left(t_{3}\right) g^{ \pm}\left(-t_{3}\right)\right], \quad(34) \\
\Sigma_{a, b}^{a(4)}(E)= & U^{4} \int_{-\infty}^{0} \mathrm{~d} t_{1} \int_{-\infty}^{\infty} \mathrm{d} t_{2} \int_{-\infty}^{\infty} \mathrm{d} t_{3} \mathrm{e}^{\mathrm{i} E t_{1}}\left[\begin{array}{c}
g^{>}\left(t_{1}\right) g^{>}\left(t_{1}-t_{2}-t_{3}\right) g^{<}\left(-t_{1}+t_{2}+t_{3}\right) \\
-g^{<}\left(t_{1}\right) g^{<}\left(t_{1}-t_{2}-t_{3}\right) g^{>}\left(-t_{1}+t_{2}+t_{3}\right)
\end{array}\right] \\
& \times\left[g^{ \pm}\left(t_{2}\right) g^{<}\left(-t_{2}\right)+g^{<}\left(t_{2}\right) g^{ \pm}\left(-t_{2}\right)\right]\left[g^{ \pm}\left(t_{3}\right) g^{<}\left(-t_{3}\right)+g^{<}\left(t_{3}\right) g^{ \pm}\left(-t_{3}\right)\right] .
\end{aligned}
$$


Moreover, figure $4 \mathrm{c}$ shows the diagram for the following terms:

$$
\begin{aligned}
& \Sigma_{c}^{r(4)}(E)=U^{4} \int_{0}^{\infty} \mathrm{d} t_{1} \int_{-\infty}^{\infty} \mathrm{d} t_{2} \int_{-\infty}^{\infty} \mathrm{d} t_{3} \mathrm{e}^{\mathrm{i} E t_{1}} \\
& \times\left[\begin{array}{l}
g^{>}\left(-t_{1}\right) g^{<}\left(t_{1}-t_{2}-t_{3}\right) g^{<}\left(t_{1}-t_{2}-t_{3}\right) \\
-g^{<}\left(-t_{1}\right) g^{>}\left(t_{1}-t_{2}-t_{3}\right) g^{>}\left(t_{1}-t_{2}-t_{3}\right)
\end{array}\right] \\
& \times\left[g^{ \pm}\left(t_{2}\right) g^{>}\left(t_{2}\right)+g^{<}\left(t_{2}\right) g^{ \pm}\left(t_{2}\right)\right]\left[g^{ \pm}\left(t_{3}\right) g^{>}\left(t_{3}\right)+g^{<}\left(t_{3}\right) g^{ \pm}\left(t_{3}\right)\right], \\
& \Sigma_{c}^{a(4)}(E)=U^{4} \int_{-\infty}^{0} \mathrm{~d} t_{1} \int_{-\infty}^{\infty} \mathrm{d} t_{2} \int_{-\infty}^{\infty} \mathrm{d} t_{3} \mathrm{e}^{\mathrm{i} E t_{1}} \\
& \times\left[\begin{array}{l}
g^{<}\left(-t_{1}\right) g^{>}\left(t_{1}-t_{2}-t_{3}\right) g^{>}\left(t_{1}-t_{2}-t_{3}\right) \\
-g^{>}\left(-t_{1}\right) g^{<}\left(t_{1}-t_{2}-t_{3}\right) g^{<}\left(t_{1}-t_{2}-t_{3}\right)
\end{array}\right] \\
& \times\left[g^{ \pm}\left(t_{2}\right) g^{>}\left(t_{2}\right)+g^{<}\left(t_{2}\right) g^{ \pm}\left(t_{2}\right)\right]\left[g^{ \pm}\left(t_{3}\right) g^{>}\left(t_{3}\right)+g^{<}\left(t_{3}\right) g^{ \pm}\left(t_{3}\right)\right] \text {. }
\end{aligned}
$$

Next, the terms brought from the diagram in figure $4 \mathrm{~d}$ are expressed by

$$
\begin{aligned}
\Sigma_{d}^{r(4)}(E)= & U^{4} \int_{0}^{\infty} d t_{1} \int_{-\infty}^{\infty} d t_{2} \int_{-\infty}^{\infty} \mathrm{d} t_{3} \mathrm{e}^{\mathrm{i} E t_{1}}\left[\begin{array}{c}
g^{>}\left(t_{1}-t_{3}\right) g^{>}\left(t_{1}-t_{2}\right) g^{<}\left(t_{2}-t_{1}\right) \\
-g^{<}\left(t_{1}-t_{3}\right) g^{<}\left(t_{1}-t_{2}\right) g^{>}\left(t_{2}-t_{1}\right)
\end{array}\right] \\
& \times g^{ \pm}\left(t_{2}\right) \operatorname{sgn}\left(t_{3}\right)\left[\begin{array}{c}
g^{>}\left(-t_{2}+t_{3}\right) g^{>}\left(t_{3}\right) g^{<}\left(-t_{3}\right) \\
-g^{<}\left(-t_{2}+t_{3}\right) g^{<}\left(t_{3}\right) g^{>}\left(-t_{3}\right)
\end{array}\right], \\
\Sigma_{d}^{a(4)}(E)= & U^{4} \int_{-\infty}^{0} \mathrm{~d} t_{1} \int_{-\infty}^{\infty} \mathrm{d} t_{2} \int_{-\infty}^{\infty} \mathrm{d} t_{3} \mathrm{e}^{\mathrm{i} E t_{1}}\left[\begin{array}{c}
g^{<}\left(t_{1}-t_{3}\right) g^{<}\left(t_{1}-t_{2}\right) g^{>}\left(t_{2}-t_{1}\right) \\
-g^{>}\left(t_{1}-t_{3}\right) g^{>}\left(t_{1}-t_{2}\right) g^{<}\left(t_{2}-t_{1}\right)
\end{array}\right] \\
& \times g^{ \pm}\left(t_{2}\right) \operatorname{sgn}\left(t_{3}\right)\left[\begin{array}{l}
g^{>}\left(-t_{2}+t_{3}\right) g^{>}\left(t_{3}\right) g^{<}\left(-t_{3}\right) \\
-g^{<}\left(-t_{2}+t_{3}\right) g^{<}\left(t_{3}\right) g^{>}\left(-t_{3}\right)
\end{array}\right] .
\end{aligned}
$$

The terms for the diagram in figure $4 \mathrm{e}$ are written by

$$
\begin{aligned}
\Sigma_{e}^{r(4)}(E)= & U^{4} \int_{0}^{\infty} \mathrm{d} t_{1} \int_{-\infty}^{\infty} \mathrm{d} t_{2} \int_{-\infty}^{\infty} \mathrm{d} t_{3} \mathrm{e}^{\mathrm{i} E t_{1}}\left[\begin{array}{c}
g^{>}\left(t_{1}-t_{2}\right) g^{>}\left(t_{1}-t_{2}\right) g^{<}\left(t_{3}-t_{1}\right) \\
-g^{<}\left(t_{1}-t_{2}\right) g^{<}\left(t_{1}-t_{2}\right) g^{>}\left(t_{3}-t_{1}\right)
\end{array}\right] \\
& \times g^{ \pm}\left(t_{2}\right) \operatorname{sgn}\left(t_{3}\right)\left[\begin{array}{c}
g^{>}\left(t_{2}-t_{3}\right) g^{>}\left(-t_{3}\right) g^{<}\left(t_{3}\right) \\
-g^{<}\left(t_{2}-t_{3}\right) g^{<}\left(-t_{3}\right) g^{>}\left(t_{3}\right)
\end{array}\right], \\
\Sigma_{e}^{a(4)}(E)= & U^{4} \int_{-\infty}^{0} \mathrm{~d} t_{1} \int_{-\infty}^{\infty} \mathrm{d} t_{2} \int_{-\infty}^{\infty} \mathrm{d} t_{3} \mathrm{e}^{\mathrm{i} E t_{1}}\left[\begin{array}{c}
g^{<}\left(t_{1}-t_{2}\right) g^{<}\left(t_{1}-t_{2}\right) g^{>}\left(t_{3}-t_{1}\right) \\
-g^{>}\left(t_{1}-t_{2}\right) g^{>}\left(t_{1}-t_{2}\right) g^{<}\left(t_{3}-t_{1}\right)
\end{array}\right] \\
& \times g^{ \pm}\left(t_{2}\right) \operatorname{sgn}\left(t_{3}\right)\left[\begin{array}{l}
g^{>}\left(t_{2}-t_{3}\right) g^{>}\left(-t_{3}\right) g^{<}\left(t_{3}\right) \\
-g^{<}\left(t_{2}-t_{3}\right) g^{<}\left(-t_{3}\right) g^{>}\left(t_{3}\right)
\end{array}\right] .
\end{aligned}
$$

Moreover, figure $4 \mathrm{f}$ denotes the diagram for the following terms:

$$
\begin{aligned}
\Sigma_{f}^{r(4)}(E)= & U^{4} \int_{0}^{\infty} \mathrm{d} t_{1} \int_{-\infty}^{\infty} \mathrm{d} t_{2} \int_{-\infty}^{\infty} \mathrm{d} t_{3} \mathrm{e}^{\mathrm{i} E t_{1}}\left[\begin{array}{c}
g^{>}\left(t_{1}-t_{3}\right) g^{>}\left(t_{1}-t_{2}\right) g^{<}\left(t_{2}-t_{1}\right) \\
-g^{<}\left(t_{1}-t_{3}\right) g^{<}\left(t_{1}-t_{2}\right) g^{>}\left(t_{2}-t_{1}\right)
\end{array}\right] \\
& \times g^{ \pm}\left(-t_{2}\right) \operatorname{sgn}\left(t_{3}\right)\left[\begin{array}{c}
g^{<}\left(t_{3}\right) g^{<}\left(t_{3}\right) g^{>}\left(t_{2}-t_{3}\right) \\
-g^{>}\left(t_{3}\right) g^{>}\left(t_{3}\right) g^{<}\left(t_{2}-t_{3}\right)
\end{array}\right], \\
\Sigma_{f}^{a(4)}(E)= & U^{4} \int_{-\infty}^{0} \mathrm{~d} t_{1} \int_{-\infty}^{\infty} \mathrm{d} t_{2} \int_{-\infty}^{\infty} \mathrm{d} t_{3} \mathrm{e}^{\mathrm{i} E t_{1}}\left[\begin{array}{c}
g^{<}\left(t_{1}-t_{3}\right) g^{<}\left(t_{1}-t_{2}\right) g^{>}\left(t_{2}-t_{1}\right) \\
-g^{>}\left(t_{1}-t_{3}\right) g^{>}\left(t_{1}-t_{2}\right) g^{<}\left(t_{2}-t_{1}\right)
\end{array}\right] \\
& \times g^{ \pm}\left(-t_{2}\right) \operatorname{sgn}\left(t_{3}\right)\left[\begin{array}{l}
g^{<}\left(t_{3}\right) g^{<}\left(t_{3}\right) g^{>}\left(t_{2}-t_{3}\right) \\
-g^{>}\left(t_{3}\right) g^{>}\left(t_{3}\right) g^{<}\left(t_{2}-t_{3}\right)
\end{array}\right] .
\end{aligned}
$$


Next, the terms formulated from diagram illustrated in figure $4 \mathrm{~g}$ are expressed by

$$
\begin{aligned}
\Sigma_{g}^{r(4)}(E)= & U^{4} \int_{0}^{\infty} \mathrm{d} t_{1} \int_{-\infty}^{\infty} \mathrm{d} t_{2} \int_{-\infty}^{\infty} \mathrm{d} t_{3} \mathrm{e}^{\mathrm{i} E t_{1}}\left[\begin{array}{c}
g^{>}\left(t_{1}\right) g^{>}\left(t_{1}-t_{2}-t_{3}\right) g^{<}\left(t_{2}-t_{1}\right) \\
-g^{<}\left(t_{1}\right) g^{<}\left(t_{1}-t_{2}-t_{3}\right) g^{>}\left(t_{2}-t_{1}\right)
\end{array}\right] \\
& \times g^{ \pm}\left(-t_{2}\right) \operatorname{sgn}\left(t_{3}\right)\left[\begin{array}{c}
g^{>}\left(t_{2}+t_{3}\right) g^{>}\left(t_{3}\right) g^{<}\left(-t_{3}\right) \\
-g^{<}\left(t_{2}+t_{3}\right) g^{<}\left(t_{3}\right) g^{>}\left(-t_{3}\right)
\end{array}\right], \\
\Sigma_{g}^{a(4)}(E)= & U^{4} \int_{-\infty}^{0} \mathrm{~d} t_{1} \int_{-\infty}^{\infty} \mathrm{d} t_{2} \int_{-\infty}^{\infty} \mathrm{d} t_{3} \mathrm{e}^{\mathrm{i} E t_{1}}\left[\begin{array}{c}
g^{<}\left(t_{1}\right) g^{<}\left(t_{1}-t_{2}-t_{3}\right) g^{>}\left(t_{2}-t_{1}\right) \\
-g^{>}\left(t_{1}\right) g^{>}\left(t_{1}-t_{2}-t_{3}\right) g^{<}\left(t_{2}-t_{1}\right)
\end{array}\right] \\
& \times g^{ \pm}\left(-t_{2}\right) \operatorname{sgn}\left(t_{3}\right)\left[\begin{array}{l}
g^{>}\left(t_{2}+t_{3}\right) g^{>}\left(t_{3}\right) g^{<}\left(-t_{3}\right) \\
-g^{<}\left(t_{2}+t_{3}\right) g^{<}\left(t_{3}\right) g^{>}\left(-t_{3}\right)
\end{array}\right] .
\end{aligned}
$$

Figure $4 \mathrm{~h}$ illustrates the diagram for the following terms:

$$
\begin{aligned}
\Sigma_{h}^{r(4)}(E)= & U^{4} \int_{0}^{\infty} \mathrm{d} t_{1} \int_{-\infty}^{\infty} \mathrm{d} t_{2} \int_{-\infty}^{\infty} \mathrm{d} t_{3} \mathrm{e}^{\mathrm{i} E t_{1}}\left[\begin{array}{c}
g^{<}\left(t_{1}\right) g^{<}\left(t_{1}-t_{2}-t_{3}\right) g^{>}\left(t_{2}-t_{1}\right) \\
-g^{>}\left(t_{1}\right) g^{>}\left(t_{1}-t_{2}-t_{3}\right) g^{<}\left(t_{2}-t_{1}\right)
\end{array}\right] \\
& \times g^{ \pm}\left(t_{2}\right) \operatorname{sgn}\left(t_{3}\right)\left[\begin{array}{c}
g^{>}\left(t_{3}\right) g^{>}\left(t_{3}\right) g^{<}\left(-t_{2}-t_{3}\right) \\
-g^{<}\left(t_{3}\right) g^{<}\left(t_{3}\right) g^{>}\left(-t_{2}-t_{3}\right)
\end{array}\right], \\
\Sigma_{h}^{a(4)}(E)= & U^{4} \int_{-\infty}^{0} \mathrm{~d} t_{1} \int_{-\infty}^{\infty} \mathrm{d} t_{2} \int_{-\infty}^{\infty} \mathrm{d} t_{3} \mathrm{e}^{\mathrm{i} E t_{1}}\left[\begin{array}{l}
g^{>}\left(t_{1}\right) g^{>}\left(t_{1}-t_{2}-t_{3}\right) g^{<}\left(t_{2}-t_{1}\right) \\
-g^{<}\left(t_{1}\right) g^{<}\left(t_{1}-t_{2}-t_{3}\right) g^{>}\left(t_{2}-t_{1}\right)
\end{array}\right] \\
& \times g^{ \pm}\left(t_{2}\right) \operatorname{sgn}\left(t_{3}\right)\left[\begin{array}{l}
g^{>}\left(t_{3}\right) g^{>}\left(t_{3}\right) g^{<}\left(-t_{2}-t_{3}\right) \\
-g^{<}\left(t_{3}\right) g^{<}\left(t_{3}\right) g^{>}\left(-t_{2}-t_{3}\right)
\end{array}\right] .
\end{aligned}
$$

Besides, the terms formulated from the diagram in figure $4 \mathrm{i}$ are written by

$$
\begin{aligned}
\Sigma_{i}^{r(4)}(E)= & U^{4} \int_{0}^{\infty} \mathrm{d} t_{1} \int_{-\infty}^{\infty} \mathrm{d} t_{2} \int_{-\infty}^{\infty} \mathrm{d} t_{3} \mathrm{e}^{\mathrm{i} E t_{1}}\left[\begin{array}{c}
g^{>}\left(-t_{1}\right) g^{<}\left(t_{1}-t_{2}-t_{3}\right) g^{<}\left(t_{1}-t_{2}\right) \\
-g^{<}\left(-t_{1}\right) g^{>}\left(t_{1}-t_{2}-t_{3}\right) g^{>}\left(t_{1}-t_{2}\right)
\end{array}\right] \\
& \times g^{ \pm}\left(t_{2}\right) \operatorname{sgn}\left(t_{3}\right)\left[\begin{array}{c}
g^{>}\left(t_{2}+t_{3}\right) g^{>}\left(t_{3}\right) g^{<}\left(-t_{3}\right) \\
-g^{<}\left(t_{2}+t_{3}\right) g^{<}\left(t_{3}\right) g^{>}\left(-t_{3}\right)
\end{array}\right] \\
\Sigma_{i}^{a(4)}(E)= & U^{4} \int_{-\infty}^{0} \mathrm{~d} t_{1} \int_{-\infty}^{\infty} \mathrm{d} t_{2} \int_{-\infty}^{\infty} \mathrm{d} t_{3} \mathrm{e}^{\mathrm{i} E t_{1}}\left[\begin{array}{c}
g^{<}\left(-t_{1}\right) g^{>}\left(t_{1}-t_{2}-t_{3}\right) g^{>}\left(t_{1}-t_{2}\right) \\
-g^{>}\left(-t_{1}\right) g^{<}\left(t_{1}-t_{2}-t_{3}\right) g^{<}\left(t_{1}-t_{2}\right)
\end{array}\right] \\
& \times g^{ \pm}\left(t_{2}\right) \operatorname{sgn}\left(t_{3}\right)\left[\begin{array}{l}
g^{>}\left(t_{2}+t_{3}\right) g^{>}\left(t_{3}\right) g^{<}\left(-t_{3}\right) \\
-g^{<}\left(t_{2}+t_{3}\right) g^{<}\left(t_{3}\right) g^{>}\left(-t_{3}\right)
\end{array}\right] .
\end{aligned}
$$

Next, the terms for diagrams denoted in figures $4 \mathrm{j}$ and $4 \mathrm{k}$ are equivalent except for the spin indices and written by

$$
\begin{aligned}
\Sigma_{j, k}^{r(4)}(E)= & U^{4} \int_{0}^{\infty} \mathrm{d} t_{1} \int_{-\infty}^{\infty} \mathrm{d} t_{2} \int_{-\infty}^{\infty} \mathrm{d} t_{3} \mathrm{e}^{\mathrm{i} E t_{1}}\left[\begin{array}{c}
g^{>}\left(t_{1}\right) g^{<}\left(-t_{1}\right) g^{>}\left(t_{1}-t_{2}-t_{3}\right) \\
-g^{<}\left(t_{1}\right) g^{>}\left(-t_{1}\right) g^{<}\left(t_{1}-t_{2}-t_{3}\right)
\end{array}\right] \\
& \times g^{ \pm}\left(t_{2}\right)\left[\begin{array}{r}
g^{ \pm}\left(t_{3}\right) g^{>}\left(t_{3}\right) g^{<}\left(-t_{3}\right) \\
+g^{<}\left(t_{3}\right) g^{ \pm}\left(t_{3}\right) g^{>}\left(-t_{3}\right) \\
+g^{<}\left(t_{3}\right) g^{>}\left(t_{3}\right) g^{ \pm}\left(-t_{3}\right)
\end{array}\right], \\
\Sigma_{j, k}^{a(4)}(E)= & U^{4} \int_{-\infty}^{0} \mathrm{~d} t_{1} \int_{-\infty}^{\infty} \mathrm{d} t_{2} \int_{-\infty}^{\infty} \mathrm{d} t_{3} \mathrm{e}^{\mathrm{i} E t_{1}}\left[\begin{array}{c}
g^{<}\left(t_{1}\right) g^{>}\left(-t_{1}\right) g^{<}\left(t_{1}-t_{2}-t_{3}\right) \\
-g^{>}\left(t_{1}\right) g^{<}\left(-t_{1}\right) g^{>}\left(t_{1}-t_{2}-t_{3}\right)
\end{array}\right] \\
& \times g^{ \pm}\left(t_{2}\right)\left[\begin{array}{c}
g^{ \pm}\left(t_{3}\right) g^{>}\left(t_{3}\right) g^{<}\left(-t_{3}\right) \\
+g^{<}\left(t_{3}\right) g^{ \pm}\left(t_{3}\right) g^{>}\left(-t_{3}\right) \\
+g^{<}\left(t_{3}\right) g^{>}\left(t_{3}\right) g^{ \pm}\left(-t_{3}\right)
\end{array}\right] .
\end{aligned}
$$


Furthermore, the terms for diagram illustrated in figure $4 \mathrm{l}$ are expressed by

$$
\begin{aligned}
\Sigma_{l}^{r(4)}(E)= & U^{4} \int_{0}^{\infty} \mathrm{d} t_{1} \int_{-\infty}^{\infty} \mathrm{d} t_{2} \int_{-\infty}^{\infty} \mathrm{d} t_{3} \mathrm{e}^{\mathrm{i} E t_{1}}\left[\begin{array}{c}
g^{>}\left(t_{1}\right) g^{>}\left(t_{1}\right) g^{<}\left(-t_{1}+t_{2}+t_{3}\right) \\
-g^{<}\left(t_{1}\right) g^{<}\left(t_{1}\right) g^{>}\left(-t_{1}+t_{2}+t_{3}\right)
\end{array}\right] \\
& \times g^{ \pm}\left(-t_{2}\right)\left[\begin{array}{c}
g^{ \pm}\left(-t_{3}\right) g^{>}\left(-t_{3}\right) g^{<}\left(t_{3}\right) \\
+g^{<}\left(-t_{3}\right) g^{ \pm}\left(-t_{3}\right) g^{>}\left(t_{3}\right) \\
+g^{<}\left(-t_{3}\right) g^{>}\left(-t_{3}\right) g^{ \pm}\left(t_{3}\right)
\end{array}\right], \\
\Sigma_{l}^{a(4)}(E)= & U^{4} \int_{-\infty}^{0} \mathrm{~d} t_{1} \int_{-\infty}^{\infty} \mathrm{d} t_{2} \int_{-\infty}^{\infty} \mathrm{d} t_{3} \mathrm{e}^{\mathrm{i} E t_{1}}\left[\begin{array}{c}
g^{<}\left(t_{1}\right) g^{<}\left(t_{1}\right) g^{>}\left(-t_{1}+t_{2}+t_{3}\right) \\
-g^{>}\left(t_{1}\right) g^{>}\left(t_{1}\right) g^{<}\left(-t_{1}+t_{2}+t_{3}\right)
\end{array}\right] \\
& \times g^{ \pm}\left(-t_{2}\right)\left[\begin{array}{c}
g^{ \pm}\left(-t_{3}\right) g^{>}\left(-t_{3}\right) g^{<}\left(t_{3}\right) \\
+g^{<}\left(-t_{3}\right) g^{ \pm}\left(-t_{3}\right) g^{>}\left(t_{3}\right) \\
+g^{<}\left(-t_{3}\right) g^{>}\left(-t_{3}\right) g^{ \pm}\left(t_{3}\right)
\end{array}\right] .
\end{aligned}
$$

\section{References}

1. Schwinger J., J. Math. Phys. (N. Y.) 1961, 2, 407.

2. Keldysh L. V., Sov. Phys. JETP, 1965, 20, 1018.

3. Kondo J., Prog. Theor. Phys., 1964, 32, 37.

4. Nozières P., J. Low Temp. Phys., 1974, 17, 31.

5. Wilson K. G., Rev. Mod. Phys., 1975, 47, 773.

6. Anderson P. W., J. Phys. C, 1970,3, 2436.

7. Nozières P., Blandin A., J. Physique, 1980,41, 193.

8. Affleck I., J. Phys. Soc. Jpn., 2005, 74, 56.

9. Ng T.-K., Lee P.A., Phys. Rev. Lett., 1988, 61, 1768; Glazman L.I., Raikh M.E., JETP Lett., 1988, 47, 452.

10. Goldhaber-Gordon D., Shtrikman H., Mahalu D., Abusch-Magder D., Meirav U., Kastner M.A., Nature, 1998, 391, 156.

11. van der Wiel W.G., De Franceschi S., Fujisawa T., Elzerman J.M., Tarucha S., Kouwenhoven L.P., Science, 2000,289, 2105.

12. Haldane F.D.M., Phys. Rev. Lett., 1978, 40, 416.

13. Cronenwett S.M., Tjerk H., Oosterkamp T.H., Kouwenhoven L.P., Science, 1998, 281, 540.

14. Yosida K., Yamada K., Prog. Theor. Phys. Suppl., 1970, 46, 244.

15. Hershfield S., Davies J.H., Wilkins J.W., Phys. Rev. B, 1992, 46, 7046.

16. Nygard J., Koehl W.F., Mason N., DiCarlo L., Marcus C. M. "Zero-field splitting of Kondo resonances in a carbon nanotube quantum dot", Preprint arXiv:cond-mat/0410467, 2004.

17. De Franceschi S., Hanson R., van der Wiel W.G., Elzerman J.M., Wijpkema J.J., Fujisawa T., Tarucha S., Kouwenhoven L.P., Phys. Rev. Lett., 2002, 89, 156801.

18. Caroli C., Combescot R., Nozières P., Saint-James D., J. Phys. C, 1971, 4, 916.

19. Lifshitz E.M., Pitaevskii L.P., Physical Kinetics (Pergamon Press, 1981).

20. Zagoskin A., Quantum Theory of Many-Body Systems (Springer-Verlag, 1998).

21. Huang K., Quantum Field Theory (John Wiley \& Sons, Inc.,1998).

22. Oguri A., J. Phys. Soc. Jpn., 2002, 71, 2969.

23. Okiji A., Fermi Surface Effects edited by Kondo J. and Yoshimori A. (Springer, 1988).

24. Yamada K., Prog. Theor. Phys., 1975, 53 970; Prog. Theor. Phys., 1976, 55, 1345.

25. Zlatić V., Horvatić B., Dolički B., Grabowski S., Entel P., Schotte K.-D., Phys. Rev. B, 2000, 63, 35104 .

26. Horvatić B., Zlatić V., Phys. Stat. Sol. (b), 1980, 99, 251.

27. Zlatić V., Horvatić B., Phys. Rev. B, 1983, 28, 6904.

28. Hewson A.C. The Kondo Problem to Heavy Fermions (Cambridge University Press, Cambridge, 1993).

29. Hamasaki M., Phys. Rev. B, 2004, 69, 115313. 


\title{
Нерівноважна теорія збурень і спектральна функція для моделі Андерсона
}

\author{
М.Гамасакі \\ Фізичний факультет університету м. Кіото, Кіото, Японія \\ Отримано 14 вересня 2006 р., в остаточному вигляді - 24 грудня 2006 р.
}

\begin{abstract}
Дана робота використовує формалізм нерівноважної теорії збурень. Отримано власні енергії до четвертого порядку теорії збурень. Доведено, що нерівноважні розвинення в дійсному часі можна пов'язати з рівноважним мацубарівським розвиненням в уявному часі. Щодо чисельних результатів, то резонанс Кондо все ще зникає для зсувної напруги, яка перевищує температури Кондо, як і було спостережено в експериментах на двох граничних системах.
\end{abstract}

Ключові слова: нерівноважна теорія збурень, нерівноважна функція Гріна, ефект Кондо, рівняння Дайсона

PACS: $71.15 .-m, 05.70 . \mathrm{Ln}$ 